\title{
Influence of mode confinement factor on the modulation properties of the Vertical Cavity Surface Emitting VCSEL laser
}

\author{
Afrah Meshal Kareem ${ }^{1}$, Younis Thanoon Younis ${ }^{2 *}$ \\ ${ }^{1,2 *}$ Department of Physics, Education College for Pure Science, University of Mosul, Mosul, Iraq \\ E-mail: ${ }^{1}$ afrah.esp116@ student.uomosul.edu.iq, ${ }^{2 *}$ younisthannon@uomosul.edu.iq
}

(Received June 29, 2021; Accepted September 05, 2021; Available online December 01, 2021)

DOI: 10.33899/edusj.2021.130566.1169, (C) 2021, College of Education for Pure Science, University of Mosul.

This is an open access article under the CC BY 4.0 license (http://creativecommons.org/licenses/by/4.0/).

\begin{abstract}
Vertical cavity surface emitting laser VCSEL is currently the main solution for many technological aspects, ultrafast optical interconnecting, Gigabit Ethernet, etc. In this paper we present the simulation results by using Optiwave ${ }^{\mathrm{TM}}$ software version 7, of the effects of optical mode confinement factor on the modulation properties (which inspected by eye diagram of the received signals) of the vertical cavity surface emitting laser VCSEL, with the range (820)Gbps of pseudo random bit sequence PRBS. The quality of the VCSEL modulation have been inspected by time domain signals, spectrums and eye diagram. Simulation results appeared an improvement in the characteristics of received bit sequences of $(8,10,12.5,16$ and 20) Gbps bit rates, represented by the rising the value of quality factor QF (1.77 to 4.81) versus increasing the value of optical mode confinement factor $\Gamma(0.2$ to 0.5$)$ respectively, and a decreasing in jitter time of superimposed traces of eye pattern and well opining eye pattern. And in the corresponding, the bit error rates BERs of the received signals have been decreased, with rising the value of mode confinement factor $\Gamma$ of the laser at constant modulation index and constant temperature of the laser. Also, the VCSEL's modulation response differences with different bitrates, causes different values of QF and BER for individual value of mode confinement factor $\Gamma$.
\end{abstract}

Keywords: Semiconductor laser, optical communication, optical modulation, VCSEL, rate equations

VCSEL تأثير معامل الحصر الضوئي على خصائص تضمين ليزر الانبعاث السطحي ذي الفجوة العمودية

$$
\begin{aligned}
& \text { افراح مشعل كريم1"، يونس ذنون يونس2 } \\
& \text { 1*32قسم الفيزياء، كلية التربية للعلوم الصرفة، جامعة الموصل، الموصل، العراق }
\end{aligned}
$$

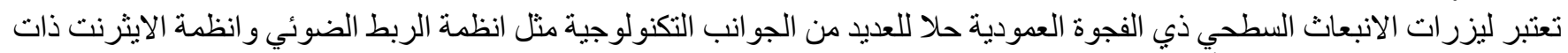

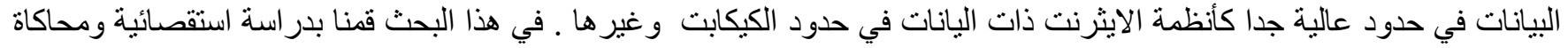

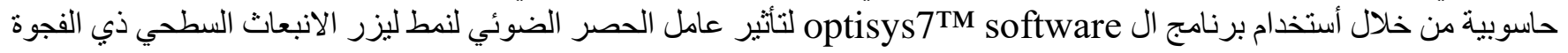

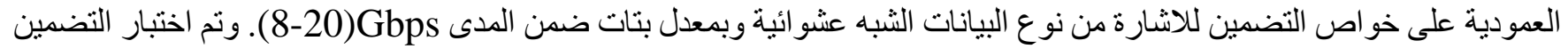

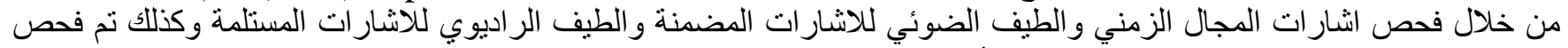

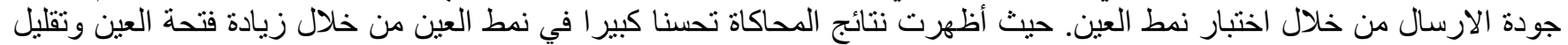

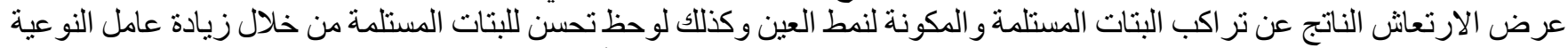

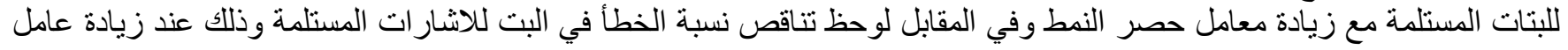

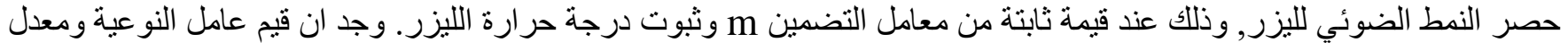




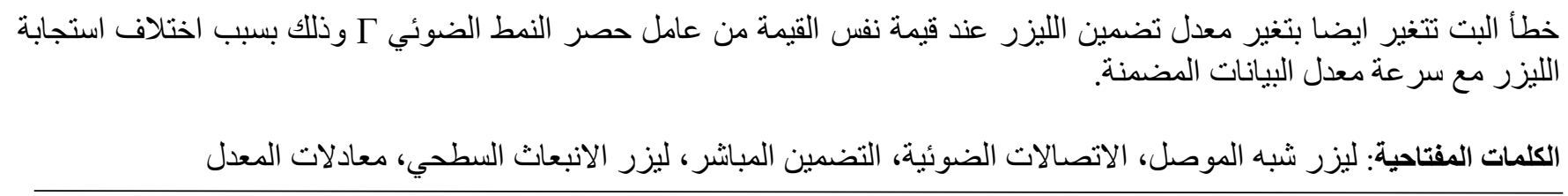

\section{Introduction}

During the past three decades, vertical cavity surface emitting lasers VCSEL, became the powerful tool in utilizing in many technology fields [1], e.g. over $10 \mathrm{~Gb} / \mathrm{s}$ data rate transceiver fiber optic communications [2][3], avionic Gigabit full duplex Ethernet [4], high resolution-fast printers [5], high performance computing (HPC) hardware [6] biomedical imaging and sensing [10]. These applications and else other, are the results of unique features of VCSEL, such as low producing costs, ultra-fast response, high temperature stability, spectral purity and other features, in comparing with the edge emitting laser(EEL). The revolutionary developments in the information technology(IT), cloud computing and cloud storage, internet of things (IOT) and the huge growth of internet users, increased the demands on expanding internet protocol (IPV6), which in turn pushed the researchers to provides ultrafast devices for processing, routing and transmission hundreds of Giga bits or Tera bits per seconds data rates and covering next generation all optical signal processing, for these reasons, VCSEL represent one of the promising solutions[5]. VCSEL modulation issue have been intensively reported[7][8][9]. Among the many parameters of VCSEL, mode confinement factor plays crucial role in modulation characteristics[11]. In this research, we proposed direct modulation of VCSEL with many Gbps bitrate of the non-return to zero(NRZ) pseudo random bit sequence PRBS signals and investigating by the simulation using Optiwave $^{\mathrm{TM}}$ software, the influence of the mode confinement factor on the modulation characteristics, e.g. on time domain signal, modulated carrier spectrum and received eye diagrams.

\section{VCSEL's dynamical Model}

The optical output of the VCSEL is governed by single mode rate equations for both carrier numbers and photon numbers, which can be written [12]

$$
\begin{aligned}
& \frac{d N}{d t}=\frac{\eta_{i} I}{e N_{w}}-(A+B N) \cdot N-G S+F_{N}(t) \\
& \frac{d S}{d t}=\Gamma \beta B N_{w} N^{2}+N_{w} G S-\frac{S}{\tau_{p}}+F_{S}(t)
\end{aligned}
$$

Where $G$ is the modal gain and it is given as:

$$
\begin{aligned}
& G=g_{0} \frac{N-N_{t}}{1+\varepsilon S} \\
& g_{0}=v_{g} \Gamma \frac{a}{V_{a c t}}
\end{aligned}
$$

(3) and $g_{0}$ is the gain coefficient, which is given by:

Where $N$ is the carrier number, $S$ is photon number, $N_{w}$ is the quantum well number, I is the bias current, $\mathrm{A}$ and $\mathrm{B}$ are the non-radiative and bimolecular-recombination coefficients respectively. $\mathrm{e}$ is the electron charge, $\eta_{i}$ is the internal quantum efficiency, $\tau_{p}$ is photon lifetime, $\beta$ is the spontaneous emission fraction coupled to output, $v_{g}$ is the group velocity, $a$ is the differential gain, $V_{a c t}$ is volume of the active layer, $N_{t}$ is carrier number at transparency, $\varepsilon$ is the nonlinear gain coefficient. Whereas the Langevin functions $F_{N}(t), F_{S}(t)$ describe the carrier and photon numbers fluctuations respectively, which are responsible for the noise intensity in the laser, their mean values are zero $\left\langle F_{N}(t)\right\rangle=\left\langle F_{S}(t)\right\rangle=0$, but they can be calculated in term of their spectral densities and in their inter-correlation product as follows:[11][13] 


$$
\begin{aligned}
& <F_{N}^{2}>=\frac{\eta_{i} I}{e}+2 g_{0} N_{w} \frac{N_{t}}{1+\varepsilon S} S \\
& <F_{S}^{2}>=2 g_{0} N_{w} \frac{N_{t}}{1+\varepsilon S} S+2 \frac{S}{\tau_{p}} \\
& <F_{N} F_{S}>=-\left[2 g_{0} N_{w} \frac{N_{t}}{1+\varepsilon S} S+\frac{S}{\tau_{p}}\right]
\end{aligned}
$$

Equations (1)to (7) are embedded in the algorithms of VCSEL component in the optiwave7 software, and they are numerically solved during circuit simulation running.

\section{3-Simulation setup}

The configuration of the transmission setup constructed from VCSEL device for which directly modulated by Non-return to zero signal which in turn driven by pseudo-random bit sequence generator (PRBG) with multi $\mathrm{Gb} / \mathrm{s}$ bitrates, as a modulated signal (or so called base band information signal). The optical output is directed via the loss free line to the photodetector as receiver, which converts optical signal to electrical one, to extract the base band modulated random bit sequences. The recovered bit sequence signal (time series) be monitored by the electrical oscilloscope visualizer(EOV), and its spectrum resolved by electrical spectrum analyzer(ESA). The strength of recovered bit sequence electrical signals is measured by power level meter in Decibel $(\mathrm{dBm})$. A part of the optical signal output from VCSEL is monitored by optical time domain visualizer(OTDV) and its spectrum is analyzed by optical spectrum analyzer(OSA), as shown in the Fig.1. The simulation is carried out by Optiwave ${ }^{\mathrm{TM}}$ software, ver.7[14]. The bias and the modulated currents of VCSEL are adjusted according to linear part of the power-current(L-I) curve of laser VCSEL, which have been predetermined as shown in the Fig.2. We have selected the values of the bias current $\mathrm{I}_{\mathrm{b}}$ and modulated signal current $\mathrm{I}_{\mathrm{m}}$ in such way that the modulation index would give out optimum values for eye diagram parameters (maximum value of quality factor QF and minimum value of bit error rate), for certain values of the VCSEL optical mode confinement factor parameter $\Gamma$, and bitrate. The parameters of VCSEL which are used in the simulation are tabulated in the Table1. Eye diagram is very helpful mean for the qualitative analysis used in fast digital transmission, it is an oscilloscope display of digital signal, recurrently sliced to get a best acting of its behavior[15]. A traditional ways to describing an eye are to measure the rise times, fall times, jitter at the middle of crossing point of the eye, transcend present and many other characteristics of eye behavior in order to make decision about measured digital signal.[16]

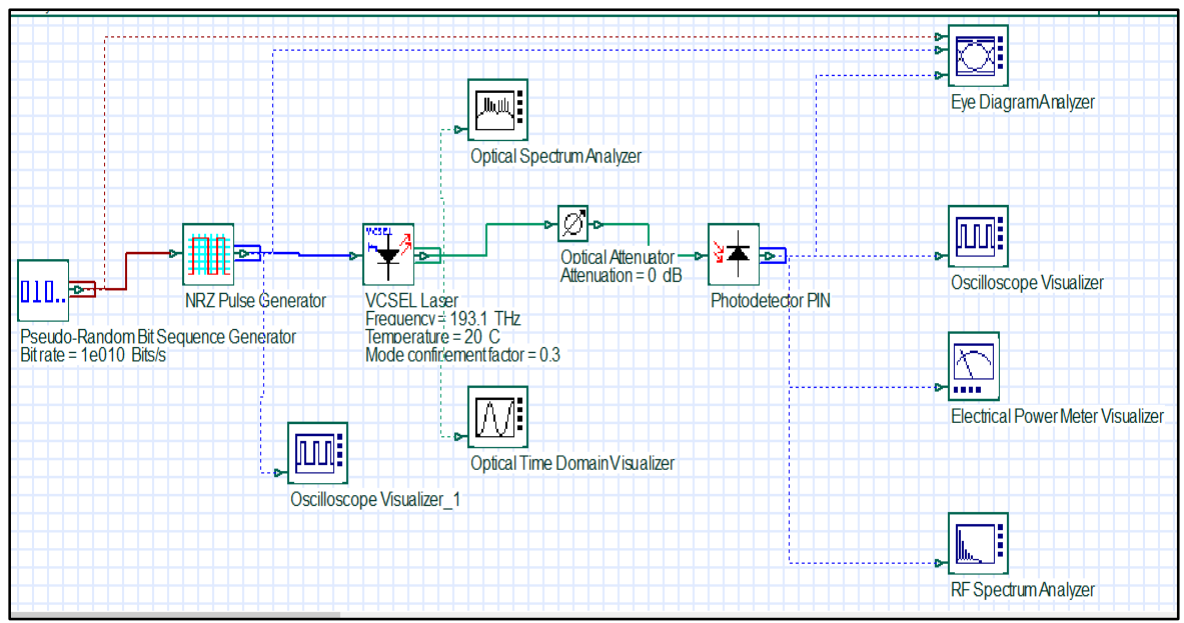

Figure 1. VCSEL optical transmitter block sets by using optiwave ${ }^{\mathrm{TM}}$ software 
Table 1. VCSEL parameters[14]

\begin{tabular}{lcc}
\hline Parameter & Value & Unit \\
\hline Active layer Volume $(\mathrm{V})$ & $1.5 \times 10^{-10}$ & $\mathrm{~cm}^{3}$ \\
Group velocity $(v)$ & $8.5 \times 10^{9}$ & $\mathrm{~cm} / \mathrm{s}$ \\
Quantum efficiency $\left(\eta_{\mathrm{Q}}\right)$ & 0.4 & \\
Differential gain coefficient $\left(\sigma_{\mathrm{d}}\right)$ & $2.5 \times 10^{-16}$ & $\mathrm{~cm}^{2}$ \\
Carrier density at transparency $\left(\mathrm{N}_{\mathrm{T}} / \mathrm{V}\right)$ & $10^{18}$ & $\mathrm{~cm}^{-3}$ \\
Mode confinement factor $(\Gamma)$ & $0.1-0.5$ & \\
Gain coefficient $(\mathrm{g})$ & 16000 & $1 / \mathrm{s}$ \\
Carrier number at transparency $(\mathrm{NT})$ & 19400000 & \\
Carrier lifetime $\left(\tau_{\mathrm{C}}\right)$ & $10^{-9}$ & $\mathrm{~s}$ \\
Photon lifetime $\left(\tau_{\mathrm{P}}\right)$ & $3 \times 10^{-12}$ & $\mathrm{~s}$ \\
Spontaneous emission factor $(\beta)$ & $3 \times 10^{-5}$ & \\
Gain compression coefficient $\left(\varepsilon_{\mathrm{m}}\right)$ & $10^{-17}$ & $\mathrm{~cm}$ \\
Linewidth enhancement factor $(\alpha)$ & 5 & \\
Current injection efficiency $\left(\eta_{\text {inj }}\right)$ & 1 & $\mathrm{w}$ \\
Scaling factor $(\mathrm{S})$ & $2.6 \times 10^{-8}$ & \\
\hline
\end{tabular}

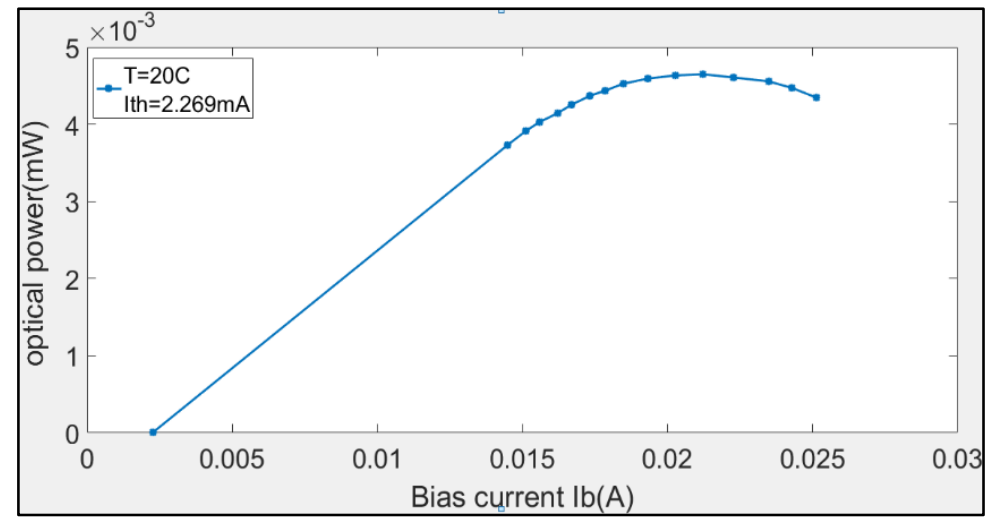

Figure 2. L-I curve of VCSEL at $20^{\circ} \mathrm{C}$ temperature[14]

\section{4- Results and Discussion}

\subsection{Simulation of VCSEL transmitter}

We have carried out the simulation of transmission of VCSEL modulation with different values of the modulated signal bit rates 8, 10,12.5, 16 and 20Gbps, such that all parameters of the VCSEL been set as in Table 1. The transmission characteristics such as modulated and optical carrier signals, their spectrums, 
received signal, spectrum, received power and eye diagram have been recorded. For the typical case of the simulation results of VCSEL's optical pulses, Fig. 3(a) shows time domain of 10Gbps NRZ format of base band signal produced by PRBS generator, monitored by the oscilloscope visualizer, while Fig 3(b) shows modulated optical carrier time domain signal and its spectrum in Fig3(c) resolved by the OSA, meanwhile Fig3(d) depicts the time domain of received signal and its showed by RF spectrum analyzer(SA)in the Fig3(e), and the Fig3(f) appears eye diagram of the received signal. There is clearly similarity between the optical pulses in Fig3(b) and received signal Fig3(d) and also between their spectrums Fig3(c) and Fig3(e). That is because of the connection between transmitter and the receiver take place via loss free transmission medium, which prevent the transmitted signals from the losses distortion.

(a)
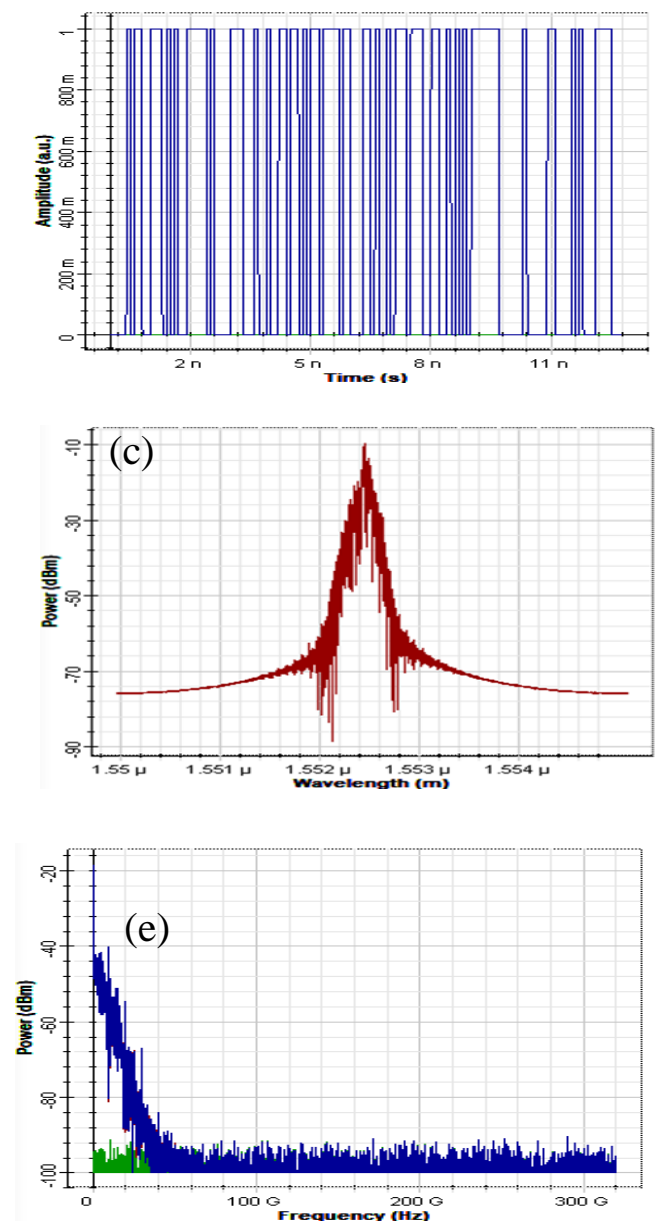

(b)
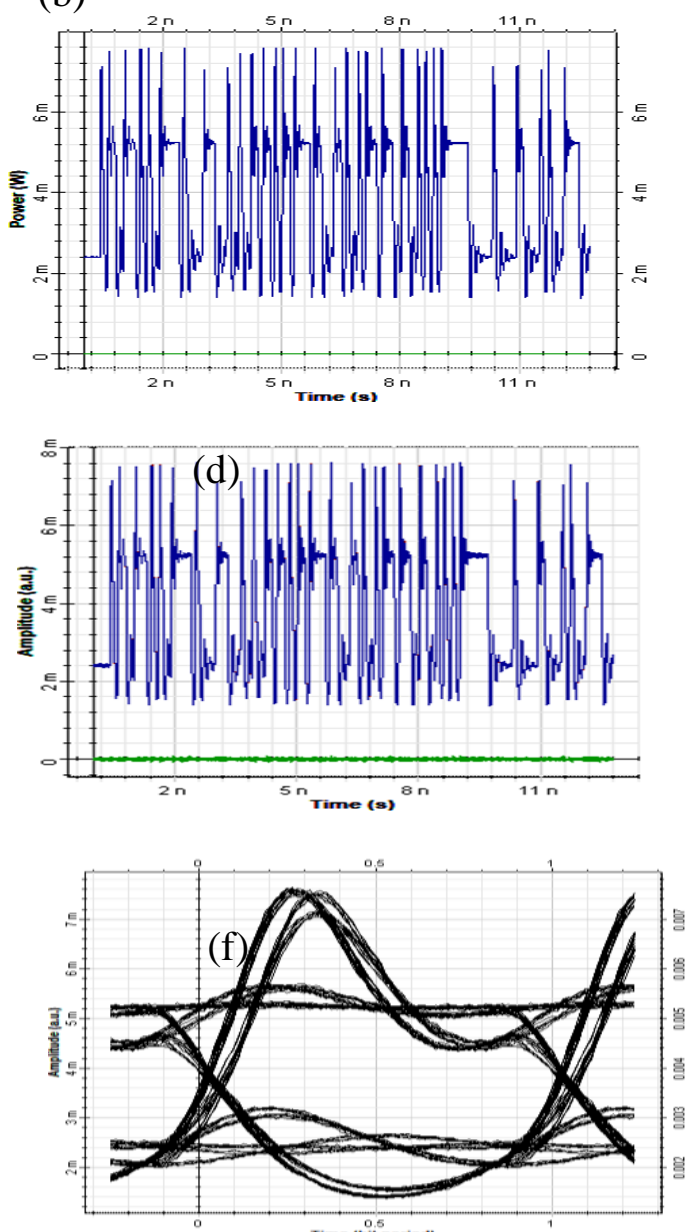

Figure 3. Modulation of VCSEL with $\Gamma=0.5$, and data rate $10 \mathrm{Gbps}$ NRZ pulses, the characteristics illustrated by: (a) baseband PRBS time series, (b) modulated optical pulses (c)spectrum of modulated optical carrier, (d) received PRBS signal, (e)RF spectrum of received signal, (f) eye pattern of received PRBS signal

\subsection{Impacts of optical confinement factor $\Gamma$ on VCSEL transmission}

In order to investigate the role of the optical confinement factor $\Gamma$ on the modulation characteristics of VCSEL, the value of $\Gamma$ is changed on each new program execution with the rest other parameters of the 
VCSEL and bitrate being fixed. The eye pattern. Fig(4) shows the influence of optical confinement factor $\Gamma$ on the eye diagram patterns, for the case of modulating VCSEL transmitter with 20Gbps NRZ pseudorandom pulses. Fig(4a) exhibits well opened eye diagram corresponds to the case of mode confinement factor $(\Gamma=0.5)$, the calculated quality factor QF is 4.81 and bit error rate BER is $7.588 \times 10^{-7}$, the bolded border of eye pattern is due to the distortion arises from Langevin noise caused by spontaneous emission which coupled with laser output[9]. Simulation results of VCSEL transmitter for the case of ( $\Gamma=$ $0.4)$ be shown in the Fig( $(4 \mathrm{~b})$, the height of eye pattern of received signal will have decreased and its border's thickness gradually increased, the evaluated QF is 3.11 and its BER is 0.00088 . The simulation results for $(\Gamma=0.3)$ appears in Fig.4(c) which displaying dense jitter of eye pattern border and the QF of the signal reduced to 2.181 and BER significantly increased to 0.0135 while the eye height drops to 0.00142 . The simulation results for the case of $\Gamma=0.2$ is given by the Fig4(d)which appear as overlapped traces, that is due to increasing of inter-symbol interference (ISI) and hence increasing the jitter and bit error rate BER to reach to 0.0349 . On the other hand, the value of the quality factor QF decreases to 1.7694.
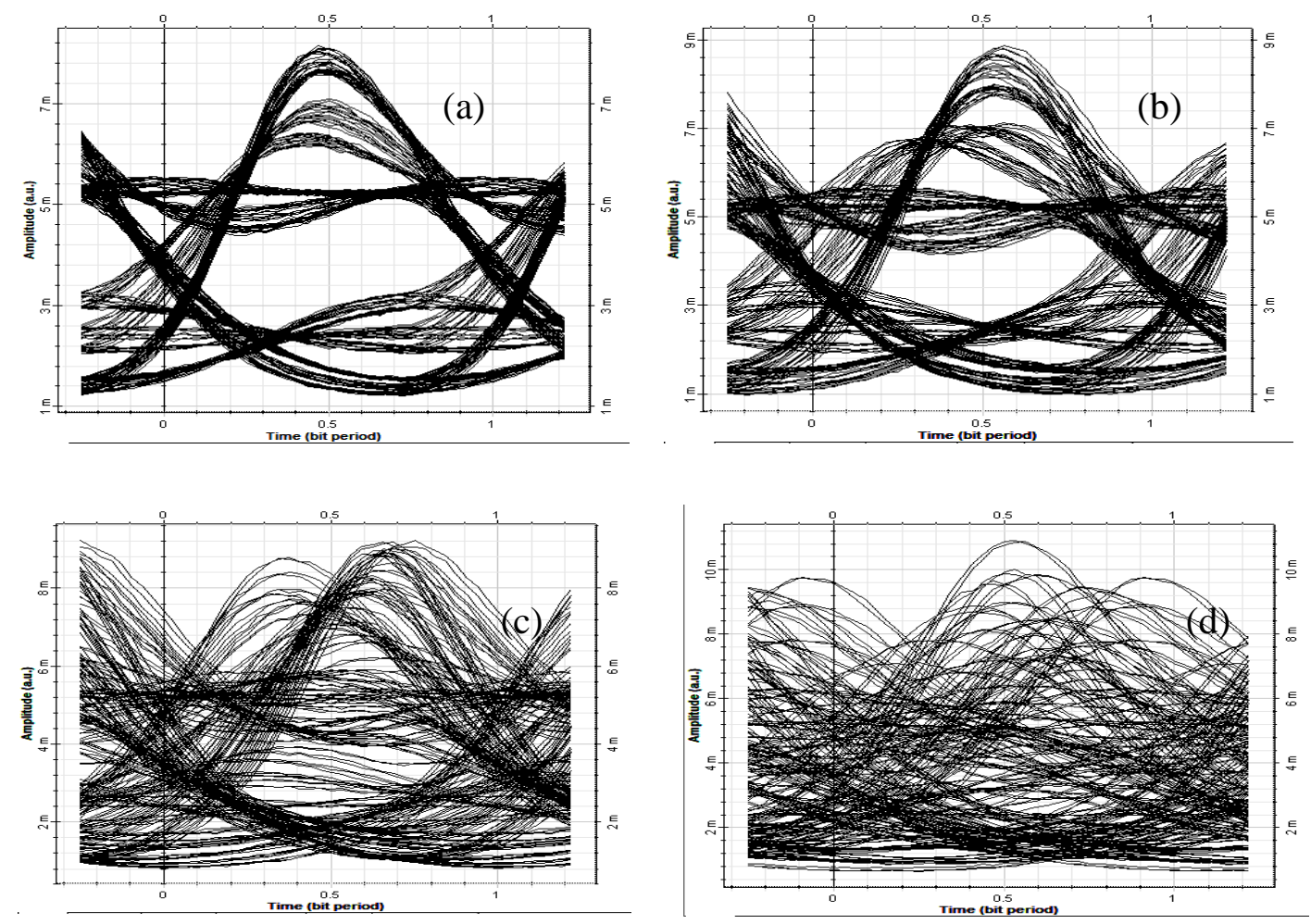

Figure 4. Effects of optical confinement factor values on Eye diagrams features for received 20Gbps NRZ format of PRBS data, modulated on VCSEL transmitter (a) $\Gamma=0.5$, $\begin{array}{lll}\text { (b) } \Gamma=0.4, & \text { (c) } \Gamma=0.3 \text { and (d) } \Gamma=0.2\end{array}$

In the previous cases of reduction modulation characteristics (decrement of QF and increasing BER) with decreasing $\Gamma$, attributed to reduction the bandwidth of VCSEL with decreasing the mode confinement factor $\Gamma$ [11]. A remarkable thing was that when the value of $\Gamma$ decreased, the strength of the received signal increased. Fig.5 explains the influence of mode confinement factor $\Gamma$ on the quality factor QF of 
the received signal for different values of data rate transmission, as it can be seen the QF increases versus raising the value of the $\Gamma$ factor. Whereas in Fig. 6 the BER of received signals reduced with increasing mode confinement factor $\Gamma$ for different data rate values. It's important to notice that the differences among the curves of Fig(5) and Fig(6), arises from differences of the laser modulation response for the different bitrates, and hence the differences in the values of QF and BER for individual value of $\Gamma$ for different values of bitrates.

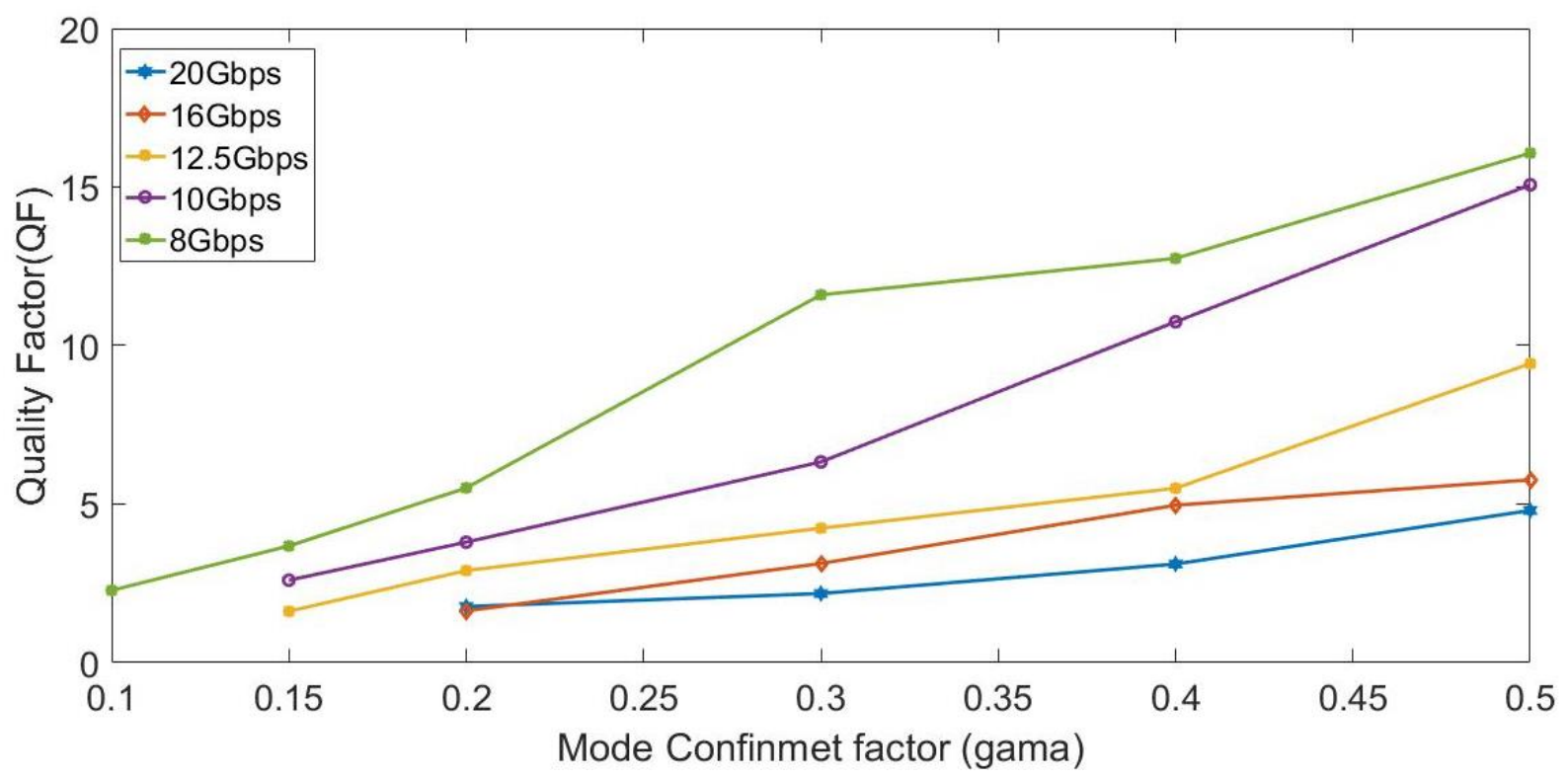

Figure 5 quality factor $(\mathrm{QF})$ of received signals as a function of mode confinement factor $\Gamma$ for different bitrates transmission

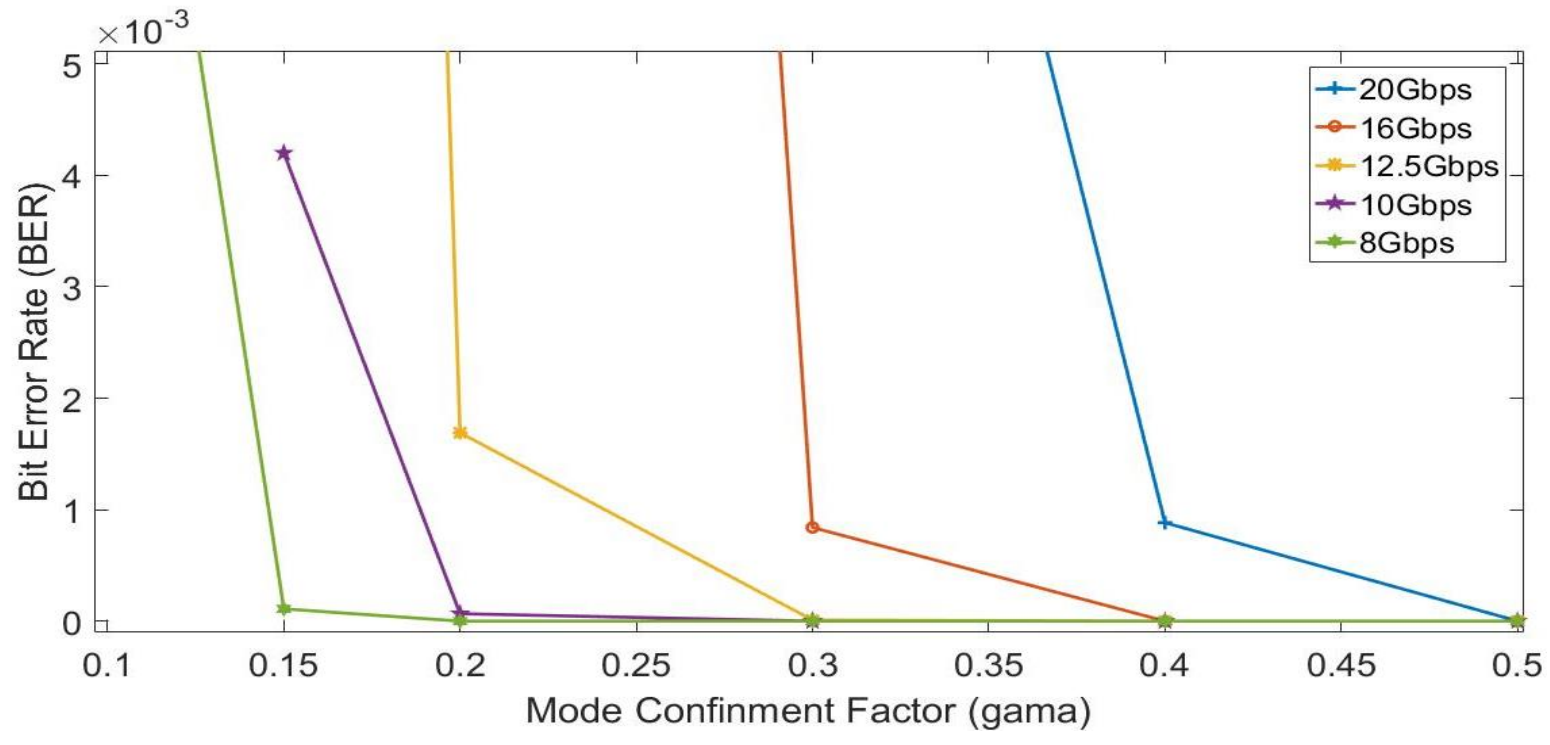

Figure 6 influence of mode confinement factor $\Gamma$ on the bit error rate BER of different bitrate transmission 


\section{Journal of Education and Science (ISSN 1812-125X), Vol: 30, No: 5, 2021 (77-85)}

\section{Conclusion}

In summary, we have investigated by the simulation of the Optiwave TM software, the role of optical mode confinement factor $\Gamma$ on the modulation characteristics (pattern of the Eye diagram), for the vertical cavity surface emitting laser VCSEL, with different values of bitrates. Eye patterns of the received signals have been highly improved by increasing mode confinement factor, especially at high data rates. This have been verified by increasing quality factor QF from 1.77 to 4.8 corresponding the increment of $\Gamma$ from 0.2 to 0.5 , with bitrate of $20 \mathrm{Gbps}$. In the same manner, increasing the value of mode confinement factor $\Gamma$ from 0.2 to 0.5 , would decrease the value of the bit error rate BER of the received data sequence, from 0.0035 to $7.6 \times 10^{-7}$. Therefore, The higher the optical confinement factor, the higher the laser modulation bandwidth and the higher frequency response speed, in addition, the modulation response differences with different bitrates, caused different values of QF and BER for individual value of mode confinement factor $\Gamma$.

\section{Acknowledgements}

The authors would like to thank the University of Mosul / Education College for Pure Science for their facilities, which have helped to enhance the quality of this work.

\section{References}

[1] K. Iga, "surface-emitting laser - its birth and generation of new optoelectronics field", ieee journal on selected topics in quantum electronics, vol. 6, no. 6, nov./dec. 2000

[2] Y. Bingliang, C. Ji, L.Wenyuan, "10Gbps vcsel driver in $0.18 \mu \mathrm{m}$ cmos technology", advanced materials research vol. 760-762 (2013) pp 147-151, trans tech publications, switzerland

[3] A. Larson, “advances in vcsels for communication and sensing”, ieee journal of selected topics in quantum electronics, vol. 17, no. 6, nov./dec. 2011

[4] K. S. Ly, A. Rissons, E. Gambardella, D. Bajon and C. Mollier, "modeling and characterization of vcsel-based avionics full duplex ethernet (afdx) gigabit links", proceedings of SPIE - the international society for optical engineering · february 2008, doi: 10.1117/12.760050

[5] M. Grabherr*, D. Wiedenmann, R. King, R. Jäger and B. Schneider, " speed it up to $10 \mathrm{~Gb} / \mathrm{s}$ and flip chip it: vcsels today", proceedings of SPIE vol. 4649 (2002) (C 2002 SPIE · 0277-786X/02

[6] A. Mutig and D. Bimberg, "progress on high-speed 980nm vcsels for short-reach optical interconnects", hindawi pub. corp., advances in optical technologies, vol. 2011, article id 290508, doi:10.1155/2011/290508

[7] T. Numai,"fundamentals of semiconductor lasers", springer-verlag ,new york, inc. 2004

[8] G. P. Agrawal and N. K. Dutta," semiconductor lasers”, 3rd printing 2001 by kluwer academic Publishers.

[9] M. F. Ahmed, A. H. Bakery and F.T. Albelady, “ digital modulation characteristics of high-speed semiconductor laser for use in optical communication systems", arab journal of science and engineering, 2013, doi 10.1007/s13369-014-1120-9 
[10] J. S. Harris, T.O’Sullivan, T. Sarmiento, M. M. Lee1 and S.Vo, "emerging applications for vertical cavity surface emitting lasers", semiconductor sci. tech.. 26 (2011) 014010 (11pp)

[11] W. Chow, G. A. Vawter, and J. Guo, "approaching intraband relaxation rates in the high-speed modulation of semiconductor lasers", ieee journal of quantum electronic, vol. 40, no. 8, august 2004

[12] A. Rissonsa, J. Perchouxa, J.C. Molliera, M. Grabherr, "noise and signal modeling of various vcsel structures", SPIE vol.5364, 2004

[13] M. Ahmed, M. Yamada, and M. Saito, "numerical modeling of intensity and phase noise in semiconductor lasers", ieee journal of quantum electronics, vol. 37, No. 12, dec. 2001

[14] A. Atiea “opti-system component library ver.7”, copyright (C) 2008 optiwave ${ }^{\mathrm{TM}}$ co. 1td., canada

[15] G. Breed," analyzing signals using the eye diagram”, high frequency electronics, copyright (C) 2005 summit technical media

[16] G. Foster, “Anatomy of an Eye Diagram”, technical notes, tektronix corp.ltd. 2010 